\title{
IMPROVING DEPOSITION TESTER TO STUDY ADHERENT DEPOSITS IN PAPERMAKING
}

M.C. Monte", M. Sánchez, A. Blanco, C. Negro and J. Tijero

Chemical Engineering Department, University Complutense of Madrid

Avda. Complutense, s/n, 28040 Madrid (Spain)

\begin{abstract}
Conventional methods used for the quantification of adherent material contained in a pulp suspension propose either filtration of the sample, which may lead to loss of sticky material in the filtrate, or dilution of the pulp, which may cause destabilization of the dissolved and colloidal material; thus, leading to unreliable results. In 1998, the Cellulose and Paper Group of University Complutense of Madrid developed a deposition tester which aimed to quantify the adherence of material (microstickies and secondary stickies from dissolved and colloidal material) that was present in white waters generated during papermaking processes. In this paper, an improved deposition tester capable of directly studying the deposition tendency of total stickies in pulps without dilution is described and validated. The design of this device prevents the rotor system from being clogged and blocked by pulps, hence, being able to quantify deposits without having to apply any filtration and/or dilution stages. In addition, the study provides determination of the equipment optimum operating conditions as well as comparison between the improved deposition tester and the one previously developed. Results show that this deposition tester can determine the adherent material contained in pulps with a consistency up to $1 \%$. The comparison of results obtained after applying both deposition quantification methods shows that the quantities of deposits that were measured with the improved tester are slightly lower than those obtained with the application of the conventional method; however, they are in the same order of magnitude. Therefore, it is possible for the improved tester to determine total stickies in all cases, including cases that it is not convenient to apply a filtration and/or a dilution stage.
\end{abstract}

Keywords: Stickies; Secondary stickies; Dissolved material; Colloidal material; Papermaking; Deposit problem

\section{Introduction}

As known, the use of recovered paper as raw material in papermaking has increased in the last years. This means that serious technical drawbacks may be introduced, especially drawbacks associated to high amounts of contaminants conveyed into the system by the recovered paper. These contaminants, together with the process chemicals used in the papermaking process, may lead to accumulation and destabilization of dissolved and colloidal material (DCM) that eventually forms deposits or stickies (Blanco et al., 2002a, 2002b; Brun et al. 2007; Ricard and Dorris, 2007a, 2007b). These deposits affect machine productivity, additive efficiency and product quality.

\footnotetext{
* Corresponding author: Tel.: + 34 913944245; Fax: + 34913944243

e-mail address: cmonte@quim.ucm.es

Address: Chemical Engineering Dept. Chemistry Falculty. Avda. Complutense s/n, 28040 Madrid (Spain)
} 
The composition of recovered paper is very heterogeneous and, therefore, the presence of contaminants in secondary fibre pulp is highly variable (Blanco et al., 1998b; Miranda et al., 2011). DCM, which is anionic, also known as anionic trash, is considered to be able to form potential deposits because, when destabilized, it can produce secondary stickies that may occur at any time in the machine, i.e., during a physic-chemical shock. The most common destabilization mechanisms are electrostatic shocks, temperature shocks and evaporative destabilization (Doshi, 1997; Monte et al., 2010; Sundberg et al., 1993).

With respect to the paper industry, determination of the contaminants present in pulps and their potential ability to deposit, along with the conditions for their destabilization, are very important parameters to be estimated especially if the high economic impact of stickies must be taken into account. Several deposition testers capable of quantifying deposits have already been proposed in the literature (Doshi et al., 2003a, 2003b). However, most of the laboratory methods, which have been applied so far to predict DCM destabilisation, have limitations, such as their low reproducibility, their failure to distinguish between sticky and non-sticky material or their need for high volume of material during the tests. In 1998, the Cellulose and Paper Group of the University Complutense of Madrid (UCM) patented a deposition tester and developed a methodology so as the potential of DCM ability to deposit was predicted (Blanco et al., 2001, 2000, 1998a; Monte, 2000). The methodology incorporated the destabilisation of DCM by polymer addition, DCM deposition on collector surfaces and the quantification of the deposits with the use of image analysis. The results of the reproducibility and their application to different systems (different adhesives, contaminants, deposit control agents and recovered paper grades) were published by Monte et al. (2004). This deposition tester has been used in several studies due to its advantages, i.e. reliability, accuracy, reproducibility, manageability, distinction between sticky and non-sticky material in suspensions and/or the possibility of extracting deposits in case further analysis is needed (Blanco et al. 2000, 2001, 2007; Doshi et al., 2003b; Monte et al., 2010; Negro et al., 1999; Tijero et al., 2011). However, this deposition tester, due to its design characteristics, could only be used with white water suspensions. Nowadays, an improved deposition tester has been developed by the UCM, a tester which is able to be used with pulps up to $1 \%$ consistency, avoid filtration and/or dilution stages, and finally estimate total stickies, and/or secondary stickies contained in pulp suspensions.

The purpose of this work was first to estimate the optimum operation conditions of the improved deposition tester by testing different pulp suspensions and then, to determine the reproducibility of the method and validate it with the aid of different samples. Finally, a comparison between the results obtained with the improved device with those taken with the traditional white water deposition tester was made.

\section{Materials and methods}

\subsection{Description of the improved deposition tester}

Similarly to the UCM deposition tester constructed for white water analysis, the equipment consists of a rotor, a collector and an axial flow propeller as shown in Figure 1. Instead of using Teflon, the rotor is now made of stainless steel and it has bigger holes on its top, bottom and side parts. The pulp suspension now enters the device 
through the top and the bottom holes and leaves it through the holes located on its side part. Also, due to the centrifugal force applied during the process, no hole blockage takes place. A surface, which is placed in front of the side holes of the equipment, allows the sample pulp to flow into it and the sticky material to deposit due to a collision mechanism. At the same time, the fluid-dynamic of the system allows the operators to promote parallel flow on the outside of the collector, which favours the formation of stickies due to a transference mechanism. The collector comprises two stainless steel coupons with a thickness of $0.05 \mathrm{~mm}$ each. These coupons are connected with the tube-support with one coupon being placed inside the tube-support and the other one being placed on the outside. The dimensions of the external stainless steel coupon are 90 × $225 \mathrm{~mm}$ and those of the internal one are $50 \times 220 \mathrm{~mm}$.

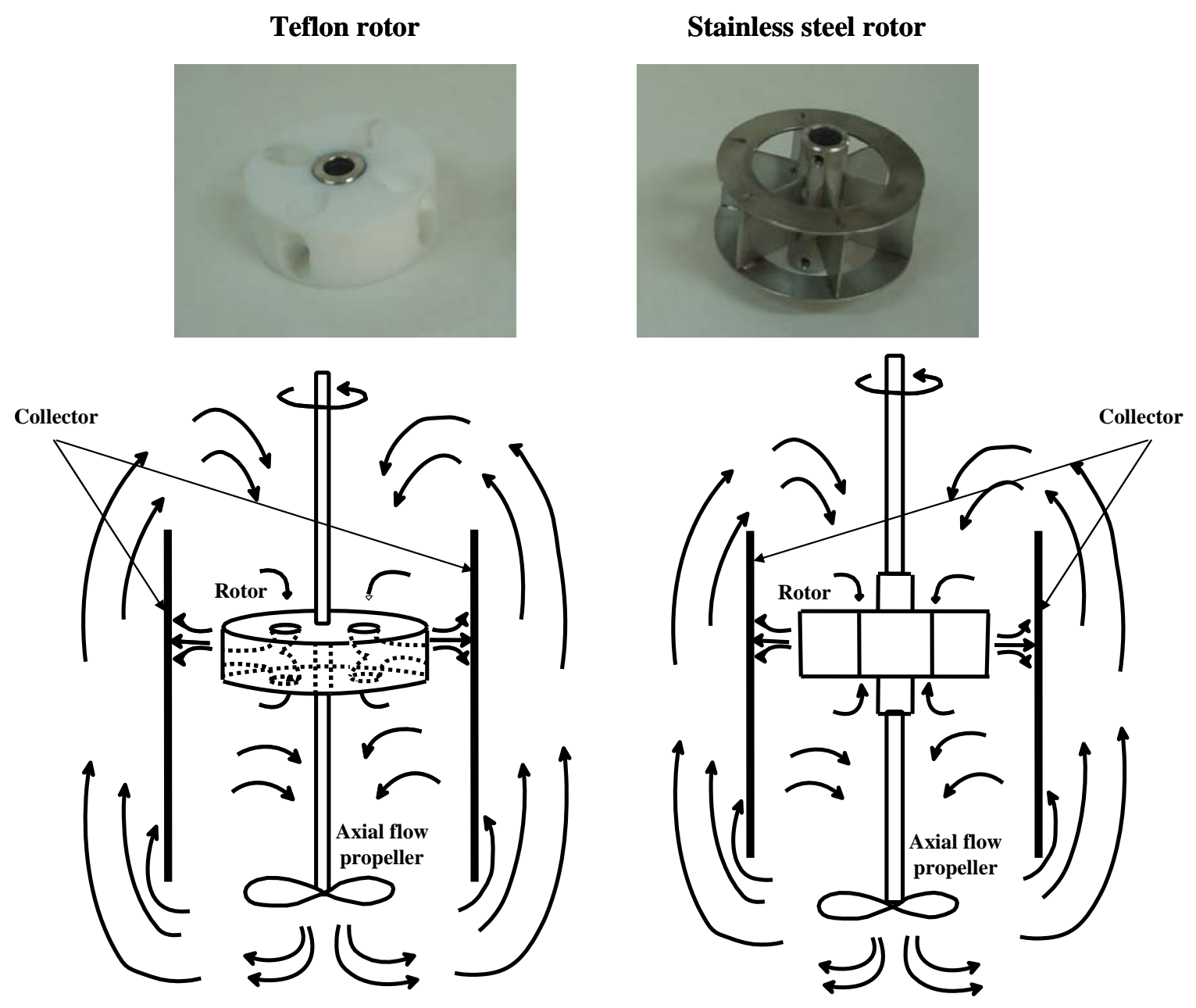

Figure 1. Comparison of designs of the two rotors and their fundamentals

When the deposition experiment is completed, the stainless steel coupons with their deposits are removed from the tube-support, rinsed with water to remove fibers and dried out in an oven at $105{ }^{\circ} \mathrm{C}$ for a few minutes. Quantitative determination of the deposits is performed by applying image analysis. The image of deposits on the coupons has been taken with a flatbed scanner HP Scanjet 6100C with an optical resolution 600 dpi. The image is then analysed by using the "Deposit Scanner v 1.2" programme, designed and constructed by the Cellulose and Paper Group of UCM as well. The programme relies on the contrast differences between the deposits and the 
background and suggests the grey level or "threshold" usually relative to the average grey scale value, at which the deposit is considered. Deposits are white and the coupons are dark, so this contrast is used to discriminate between the collector surface and the deposits. Results are as follows: area covered by deposits in $\mathrm{mm}^{2}, \mathrm{~mm}^{2}$ of deposits per $\mathrm{m}^{2}$ of surface (ppm) and percentage of covered area of deposits (\%).

\subsection{Methodology for sample preparation}

By using the improved tester, it is not necessary to filter the sample in order to obtain white water or to dilute it to avoid plugging of the rotor holes. If necessary, the raw materials are disintegrated at the desired consistency and the pulp obtained is tested with the improved tester. If secondary stickies must be studied, the cationic demand of the supernatant of the pulp after its centrifugation has to be measured, as with the tester for white waters. The cationic demand of waters is measured by applying colloidal titration with the aid of an automatic titrator Crison connected with a particle charge detector, Mütek PCD 03 (BTG). This particle charge detector can indicate the final stage of titration, which occurs when the isoelectric point has been reached. The concentration of the polymer solution used is approximately $0,0015 \mathrm{~N}$ with polyethylene-imine (PEI) of high molecular weight $\left(1.3^{*} 10^{3}\right)$ and medium-high cationic charge (4.2 meq $\mathrm{g}^{-1}$ ) (Polymin SK, BASF) being used as the polymer.

\subsection{Operational conditions of the improved deposition tester}

The main operational variables are the experimentation time and the rotor speed, variables which are able to determine the amount of deposits. These parameters were optimized, with their optimisation being based on the operational conditions of the deposition tester for white waters. Short experimental times and/or too low agitation speeds would produce small quantities of deposits. On the contrary, long experimental times would produce excessive amounts of deposits that could fall off the collector. Excessively high turbulence would remove the deposits from the surface, in both cases mentioned above producing at the same time small quantities of deposits. Initial experiments, using pulps at two concentrations or different consistencies (0.5 and 1\%), at different times and at different agitation speeds were performed to make a compromise and determine the optimum operating conditions for each consistency. Deposition temperature was fixed to $50^{\circ} \mathrm{C}$ as it is a typical temperature value for pulp suspensions in paper mills and is well above the softening temperature of synthetic polymers so deposition on the collector surfaces is favoured. The pulps used were prepared by using a Eucaliptus Globulus bleached pulp containing $0.5 \%$ of commercial adhesive as a source of potential stickies. The optimum conditions that were obtained for each consistency are summarized and compared with values provided by the deposition tester for white waters in Table 1. For a time of 60 minutes, the agitation speed has to be higher when the consistency increases so that hole plugging can be avoided. 
Table 1. Operating conditions of deposition testers

\begin{tabular}{lccc}
\hline & $\begin{array}{c}\text { Deposition tester for } \\
\text { white waters }\end{array}$ & \multicolumn{2}{c}{$\begin{array}{c}\text { Deposition tester for } \\
\text { pulps }\end{array}$} \\
\hline Consistency (\%) & $<0,01$ & 0,5 & 1 \\
\hline Agitation speed (rpm) & 250 & 300 & 400 \\
\hline Time (min) & 60 & 60 & 60 \\
\hline Temperature $\left(\mathbf{(}^{\circ} \mathbf{C}\right)$ & 50 & 50 & 50 \\
\hline Volume (mL) & 1800 & 1800 & 1800 \\
\hline
\end{tabular}

\subsection{Repeatability experiments}

As known, repeatability is defined as the value below which the absolute difference between two single test results that have been obtained after having applied the same method and having used the same material under the same conditions (operators, apparatus, laboratory and/or a short interval of times) are expected to match. The repeatability of the method was estimated based on the error of the experiments calculated statistically from the confidence interval (CI) using expression (1). The CI was determined by both the arithmetic mean value and the standard deviation for a confidence level of $95 \%(\alpha=0.05)$ with equation (2) (Monte et al., 2004).

$$
\varepsilon(\%)=\frac{|(\bar{X} \pm C I)|-\bar{X}}{\bar{X}} \times 100=\frac{| \pm C I|}{\bar{X}} \times 100
$$

where:

$\bar{X}$ : Mean value

CI: Confidence interval

$$
\mathrm{CI}= \pm \mathrm{t} \times \frac{\sigma}{\sqrt{\mathrm{n}-1}}
$$

where:
CI: Confidence interval
t: t-Student for confidence level of $95 \%$ and $(n-1)$ degrees of freedom.
$\sigma:$ Standard deviation of the sample
$\mathrm{n}$ : Size of the sample

The repeatability was estimated at pulp consistencies of $0.5 \%$ and $1 \%$. A set of twelve deposition experiments using pulps prepared from Eucaliptus Globulus bleached pulp which contained $0.5 \%$ of a commercial adhesive as a source of potential stickies, were disintegrated at the consistency that had to be studied. The stickies deposited on coupons were measured by using an image analysis system.

\subsection{Raw materials for method validation}

The validation of the improved deposition tester was made by using the methodology mentioned above in two cases:

1. Study of the contaminant concentration contained in a pulp suspension. In this study, Eucaliptus Globulus bleached pulp suspensions at different concentrations of 
adhesive were used. The source of adhesive was labels with a concentration of $20 \%$ of a hydrodispersable adhesive, an acrylic-based adhesive.

2. Study of the ability to deposit of a deinking pulp from a paper mill. In this study, mixtures of a deinking pulp (DIP) from the inlet of the second flotation stage of a German deinking line producing DIP for newsprint production (dirty pulp) with a bleached beech sulphite pulp (clean pulp) were used.

\section{Results and discussions}

\subsection{Repeatability experiments}

The results of the total deposits $\left(\mathrm{mm}^{2}\right.$ of deposits on the internal coupon plus $\mathrm{mm}^{2}$ of deposits on the external coupon) from repeatability experiments, together with the error for both $0.5 \%$ and $1 \%$ consistencies, are summarized in Table 2 . It can be observed that the error of the method applied was smaller than $10 \%, 4.8 \%$ and $6.2 \%$, respectively.

Table 2. Total microstickies and error of the improved deposition tester for different consistencies

\begin{tabular}{lcc}
\hline Consistency (\%) & 0,5 & 1 \\
\hline Total stickies mean $\left.\mathbf{( m m}^{2}\right)$ & 529 & 676 \\
\hline Error (\%) & 4,8 & 6,2 \\
\hline
\end{tabular}

\subsection{Method validation}

The validation of the improved deposition tester was made by using the methodology mentioned above, which studied the formation of deposits at two different consistencies, namely at $0.5 \%$ and $1 \%$ with:

- Pulp suspensions at different concentrations of contaminants (adhesive), and

- Mixtures of a DIP from a paper mill with a clean pulp.

\subsubsection{Study of the adhesive concentration contained in a pulp suspension}

The experiments were conducted after disintegrating an Eucaliptus Globulus bleached pulp at $0.5 \%$ and $1 \%$ consistency, respectively. Adhesive labels were used as the source of dissolved and colloidal material until pulps with an adhesive concentration between 0 and $5 \%$ were obtained. Total stickies and secondary stickies were obtained by using the new deposition tester.

\section{Total stickies}

The results of the total stickies at consistencies of $0.5 \%$ and $1 \%$ are shown in Figures 2 and 3 , respectively. 


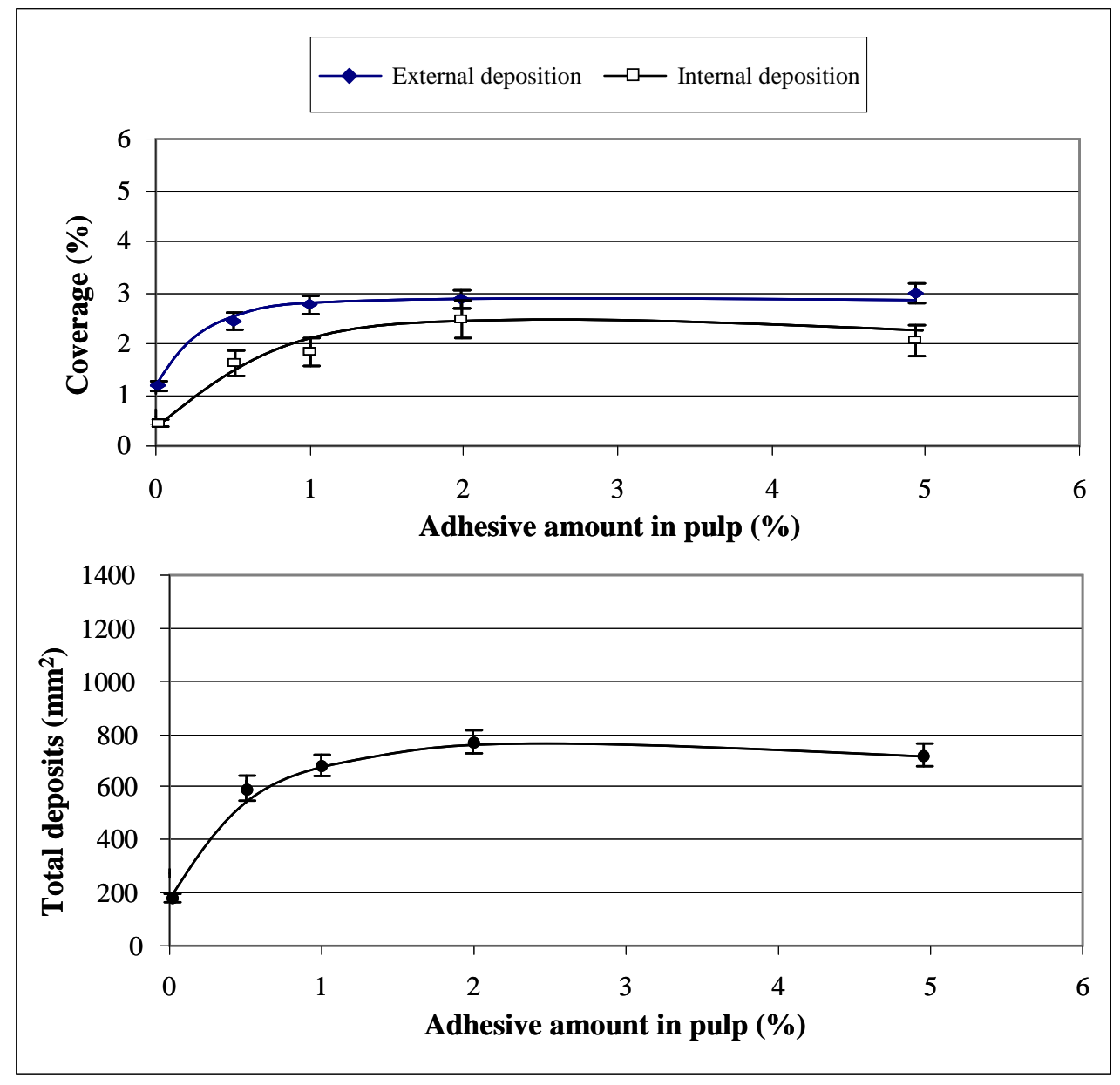

Figure 2. Effect of adhesive concentration on the deposit formation at $0.5 \%$ consistency 


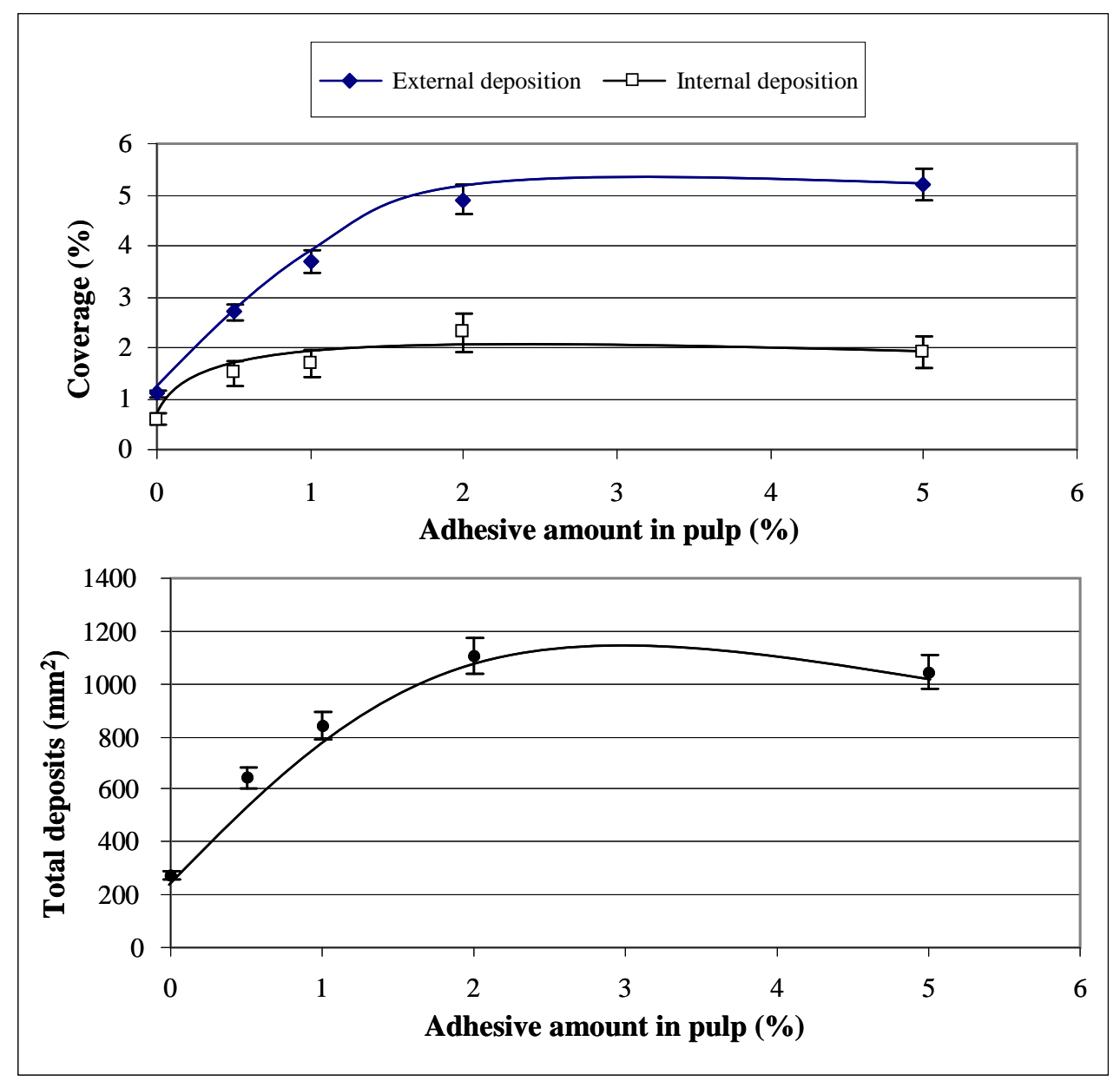

Figure 3. Effect of adhesive concentration on the deposit formation at $1 \%$ consistency

From Figures 2 and 3, it can be observed that for both consistencies, the amount of the deposits increased each time the amount of adhesive in the pulp increased as well. Also, the amount of deposits increased each time the consistency of pulp increased as well, and that was due to the increased amount of the adhesive that was present in the higher consistency pulp. Therefore, it can be observed that the amount of deposits measured above $2 \%$ adhesive concentration for both consistencies is similar. This may be due to the image analysis system only detects the deposits in two dimensions and, although more deposits are collected at high adhesive concentration, there is a limit from which the amount detected does not increase when the amount of deposit increases.

As an example, Figure 4 shows the image of the coupons with deposits obtained at 1\% consistency pulp. 


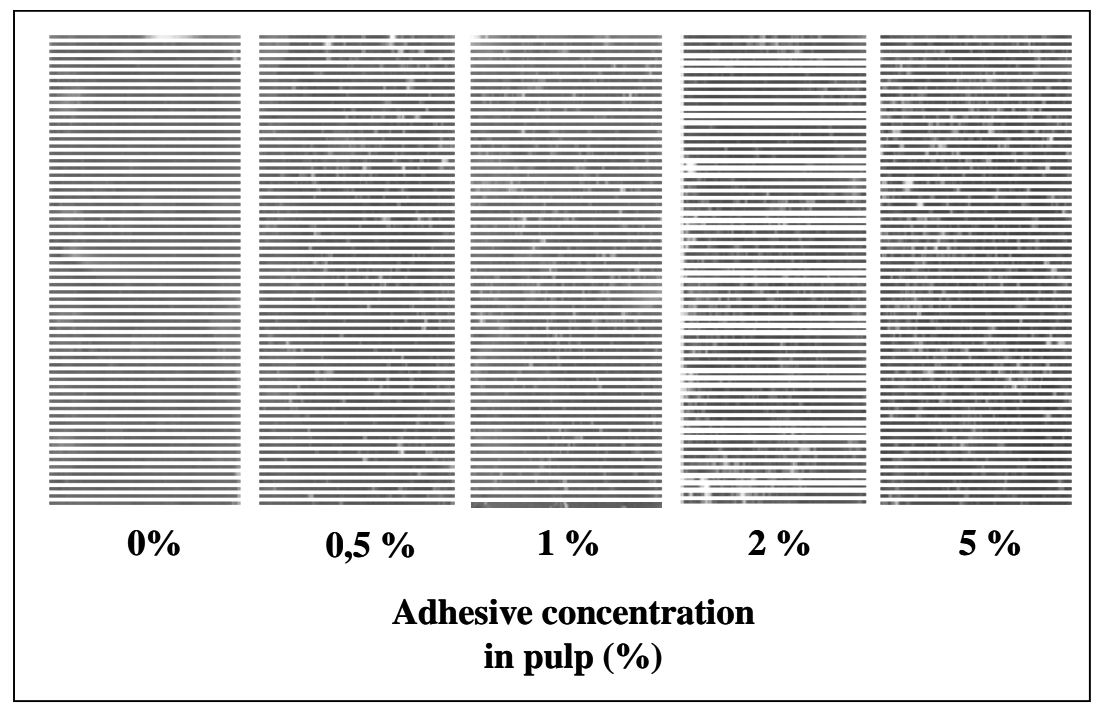

Figure 4. Deposits obtained at 1\% consistency pulp

\section{Secondary stickies}

Secondary stickies were measured by destabilizing the DCM contained in the pulp with the addition of PEI as a destabilization cationic agent. The amount of PEI was calculated from the measurement of the cationic demand of the supernatant after the centrifugation of a pulp sample. The results that were taken when consistencies changed from $0.5 \%$ to $1 \%$ in the pulps are presented in Figures 5 and 6, respectively. Also in this case, it can be observed that the amount of secondary stickies detected increase slightly above $2 \%$ adhesive concentration for both consistencies due to the image analysis system. 


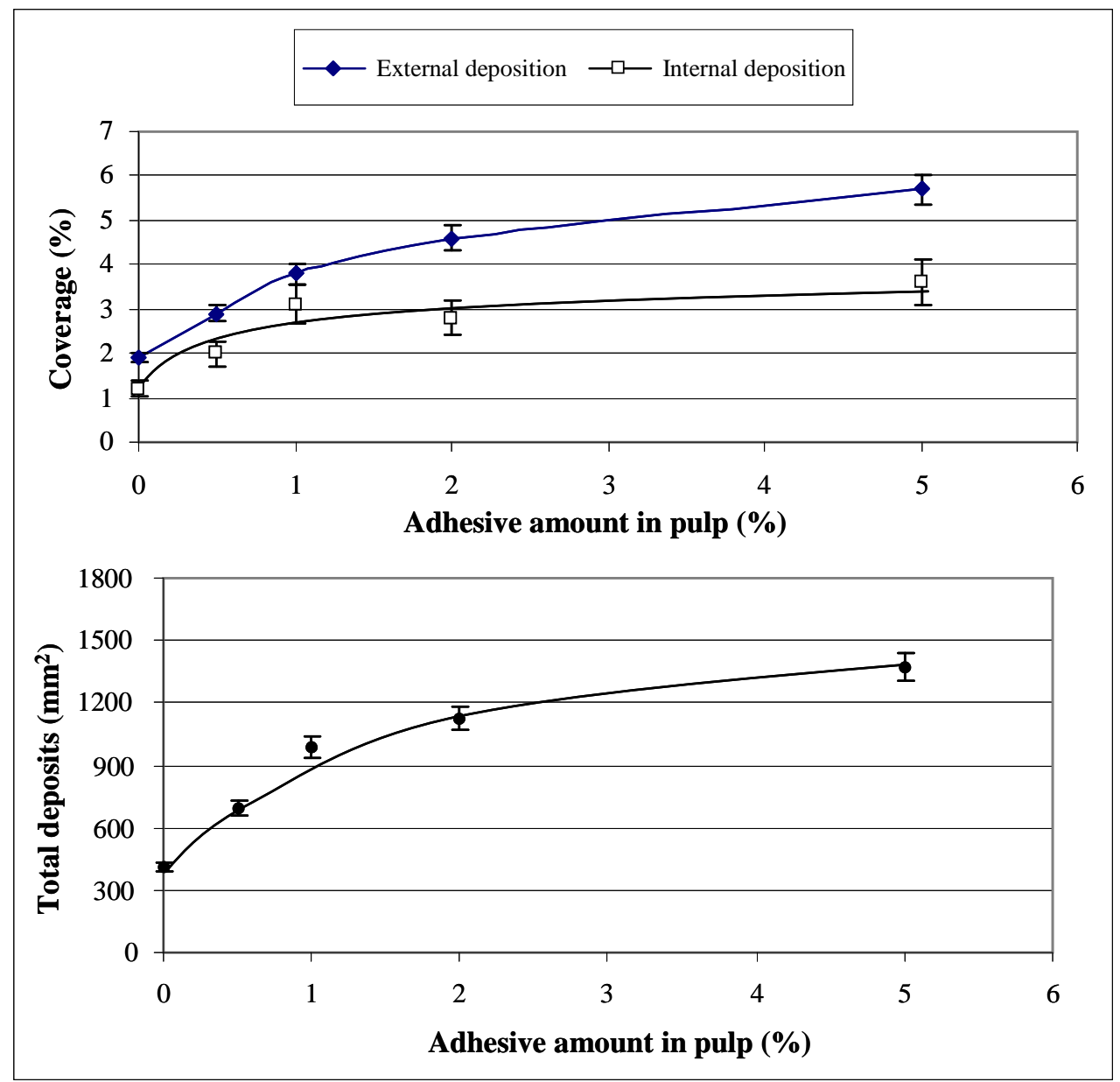

Figure 5. Effect of adhesive concentration on the secondary stickies formation at $0.5 \%$ consistency. 


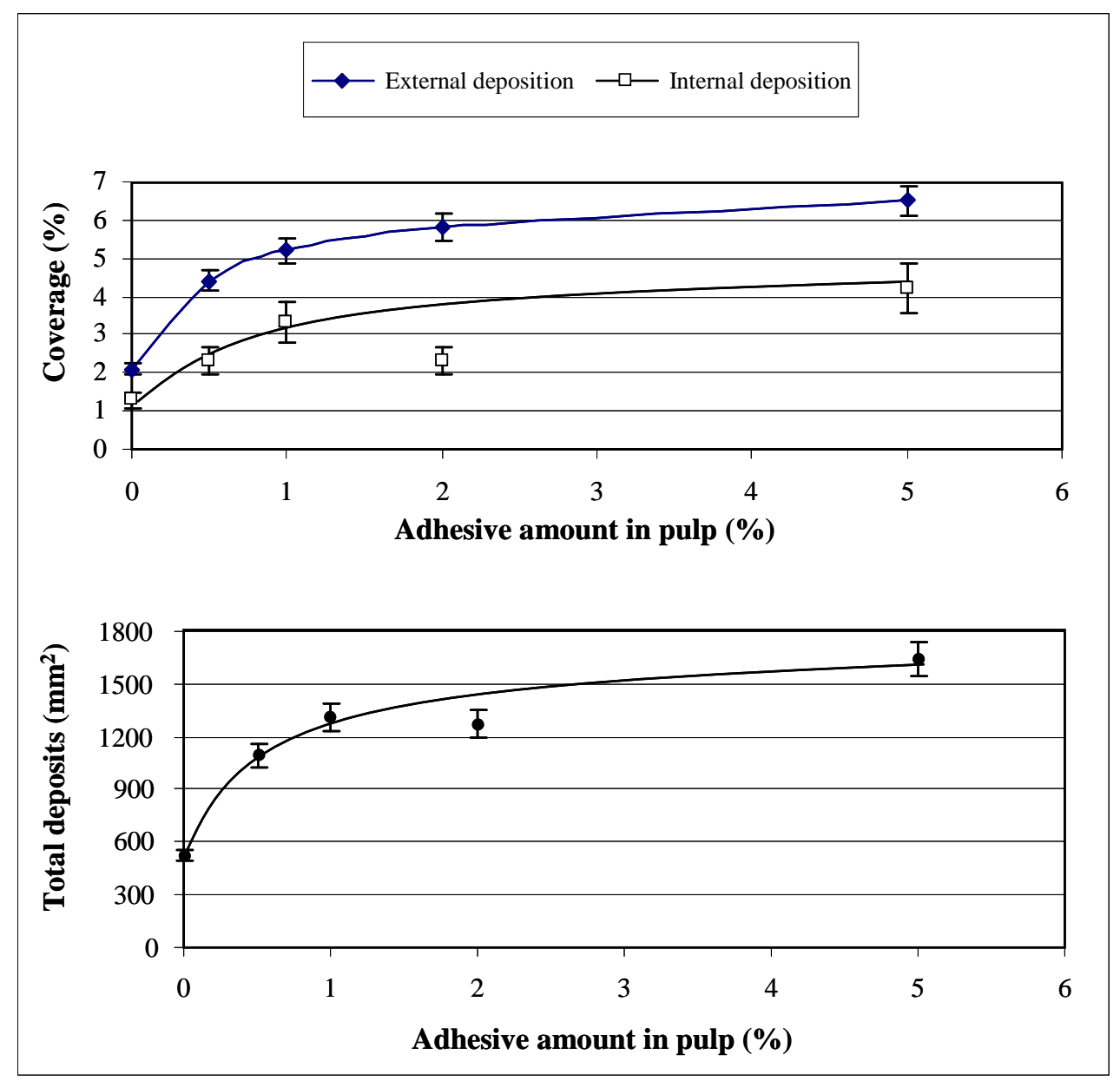

Figure 6. Effect of adhesive concentration on the secondary stickies formation at $1 \%$ consistency

The applied trend lines show that an increase in the secondary stickies occurs, when the amount of adhesive in the pulp also increases. Finally, the amounts of secondary stickies that were formed at $1 \%$ consistency are larger than those formed at $0.5 \%$ consistency. That was due to the fact that a larger amount of DCM was present at a higher consistency.

\subsubsection{Study of the ability to deposit of a deinking pulp from a paper mill}

To further validate the improved deposition tester, a set of three experiments were conducted. Throughout the experiments clean pulp mixed with pulp from the inlet of the second flotation stage of a German deinking line producing DIP for newsprint production (dirty pulp) obtaining mixtures with a consistency of 1\%. Figure 7 shows the results of stickies and secondary stickies. In this case, as expected, the deposits increase when the amount of contaminant in the sample increases.

The results obtained validate the new deposition tester to measure the depositability of the pulp suspensions. 


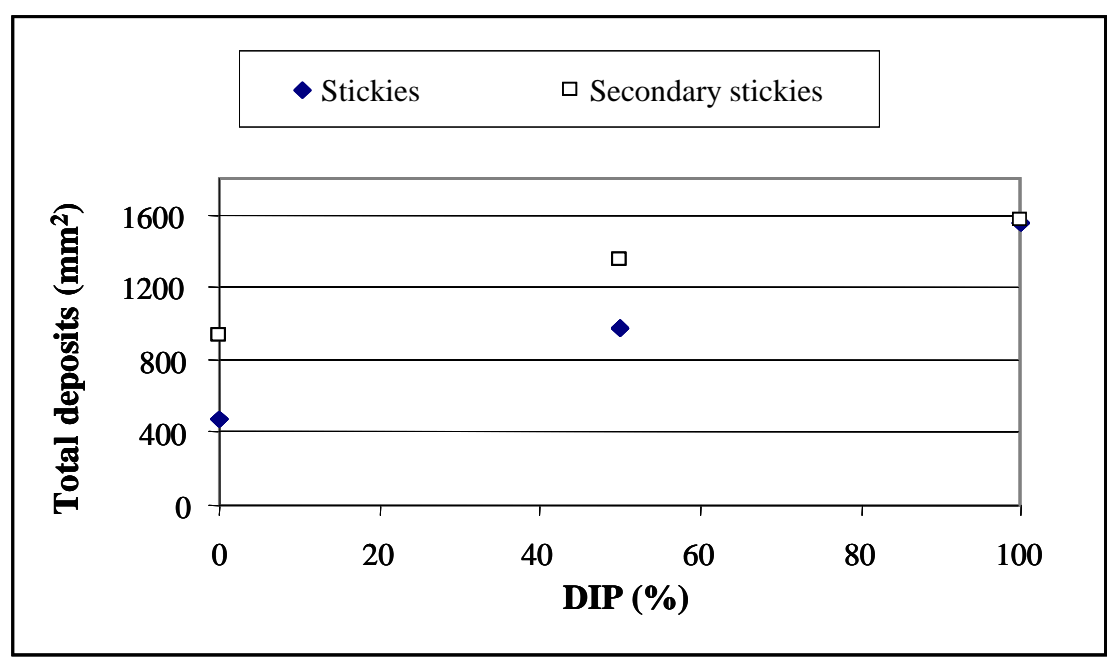

Figure 7. Effect of contaminant concentration on the deposit formation at $1 \%$ consistency

\subsection{Comparison between the improved deposition tester and the white water deposition tester}

To compare the results taken both for white waters and for pulp suspensions, series of different experiments were carried out.

\subsubsection{Comparison when synthetic samples are used}

Pulp suspensions were prepared at $0.5 \%$ and $1 \%$ consistencies, adding as contaminant $0.5 \%$ of a commercial based-acrylate adhesive. These experiments were conducted following the protocol described in section 2.2, and they successfully determined the contents of stickies and secondary stickies provided by both testers. With respect to white water experiments, pulps were filtered through a dynamic drainage jar (DDJ) and tested with the white water deposition tester. In Figures 8 and 9, it is shown a comparison between the results taken after using both testers at different pulp consistencies. In general, for both consistencies, the amount of deposits obtained using the tester for white waters is larger than the one obtained when using the tester for pulps. The difference is even bigger in the case of secondary stickies due to the fact that the destabilization agent (PEI) added to the pulp is acting as a fixing agent, which makes DCM stick on the fibres, then, avoiding any deposit formation on the collectors, together with any formation of secondary stickies. On the other hand, the quantity of stickies obtained when the tester for pulps was used is slightly smaller, as trapping of the sticky particles within the fibres may occur, so deposition due to stearic impediment is avoided. 


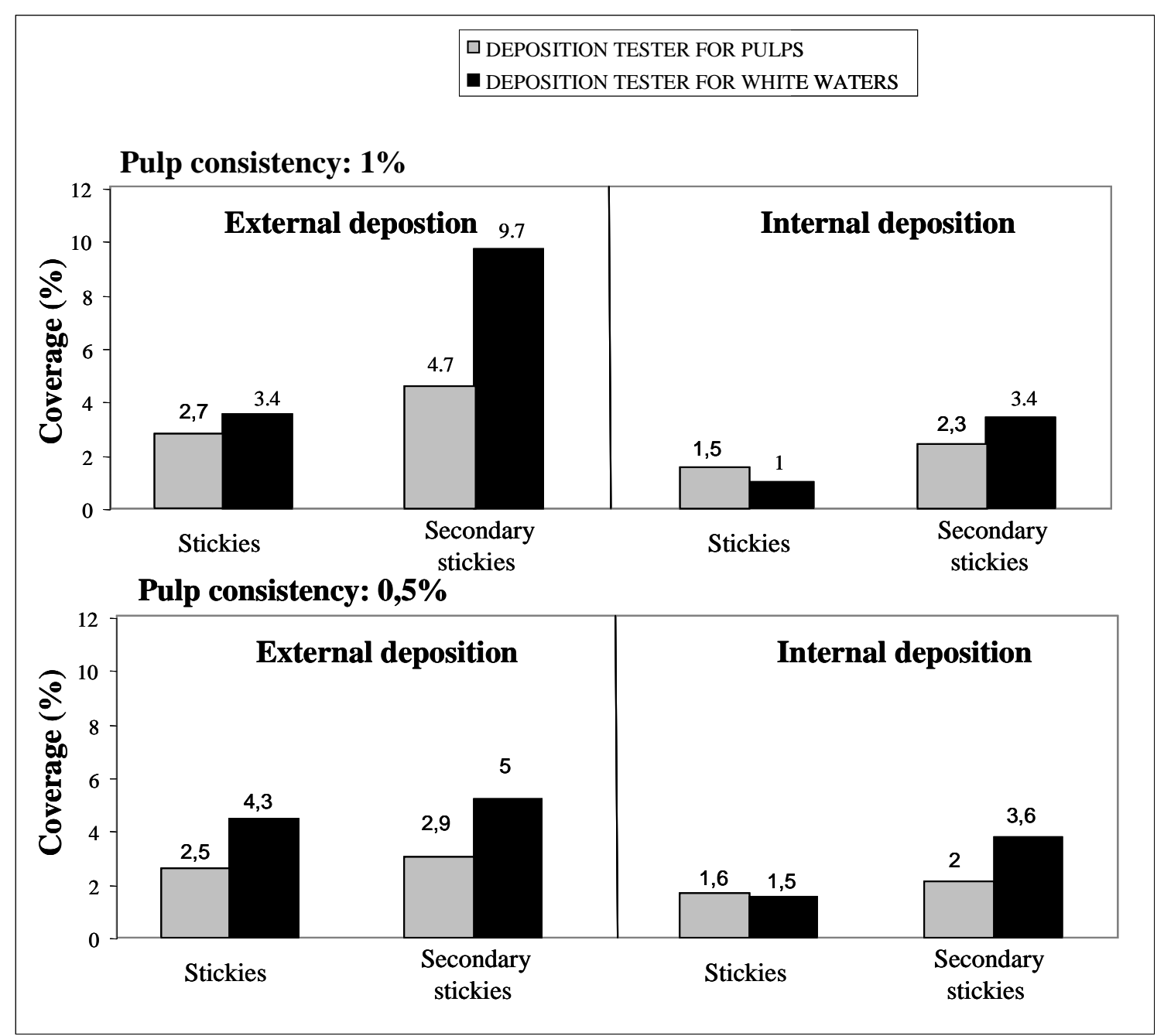

Figure 8. Comparison of the external and internal deposition obtained by both rotors at different pulp consistencies 


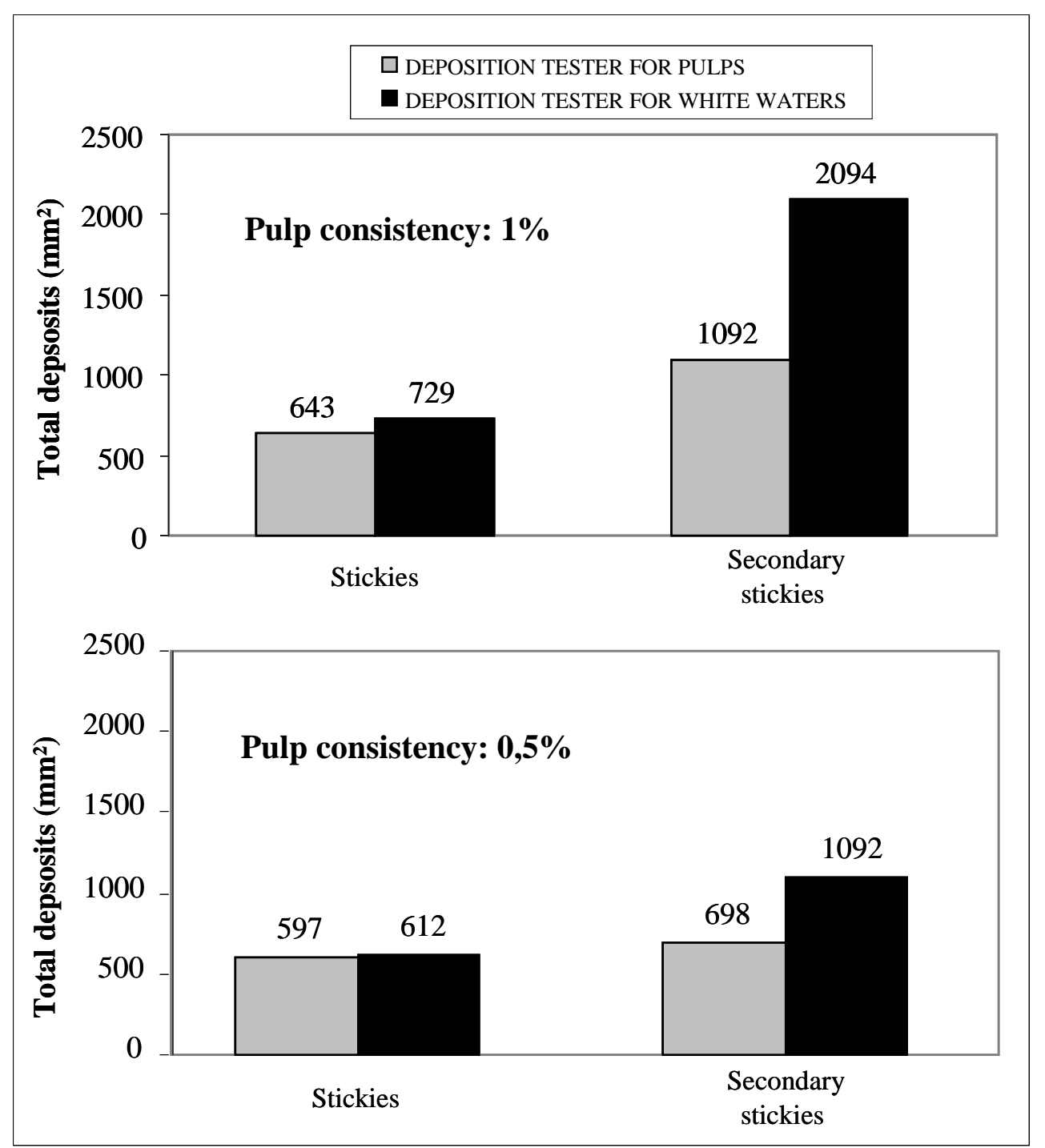

Figure 9. Comparison of the total deposits obtained by both testers at different pulp consistencies

\subsubsection{Comparison when industrial samples are used}

Experiments with both deposition testers were performed, using as sample a pulp from the inlet of the second flotation stage of a German deinking line producing DIP for newsprint production with a consistency of $1 \%$. Regarding the device for white waters, the sample was filtered according to the process shown in Figure 2. The results of the external and internal deposition after using both testers, together with their total amounts of deposits are shown in Figures 10 and 11. As seen in Figures 10 and 11, the predicted trend lines are similar to those predicted when the synthetic samples were used. 


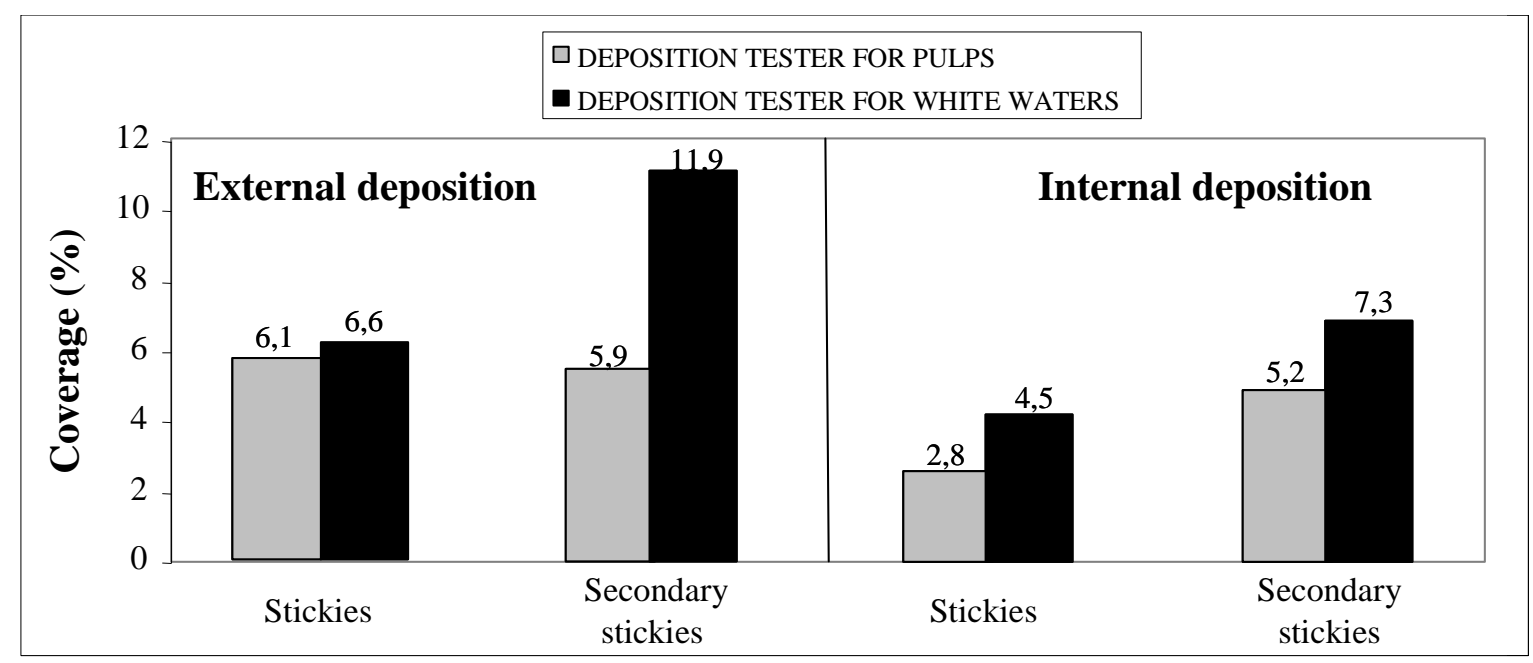

Figure 10. Comparison of the external and internal deposition obtained by both deposition testers using an industrial sample

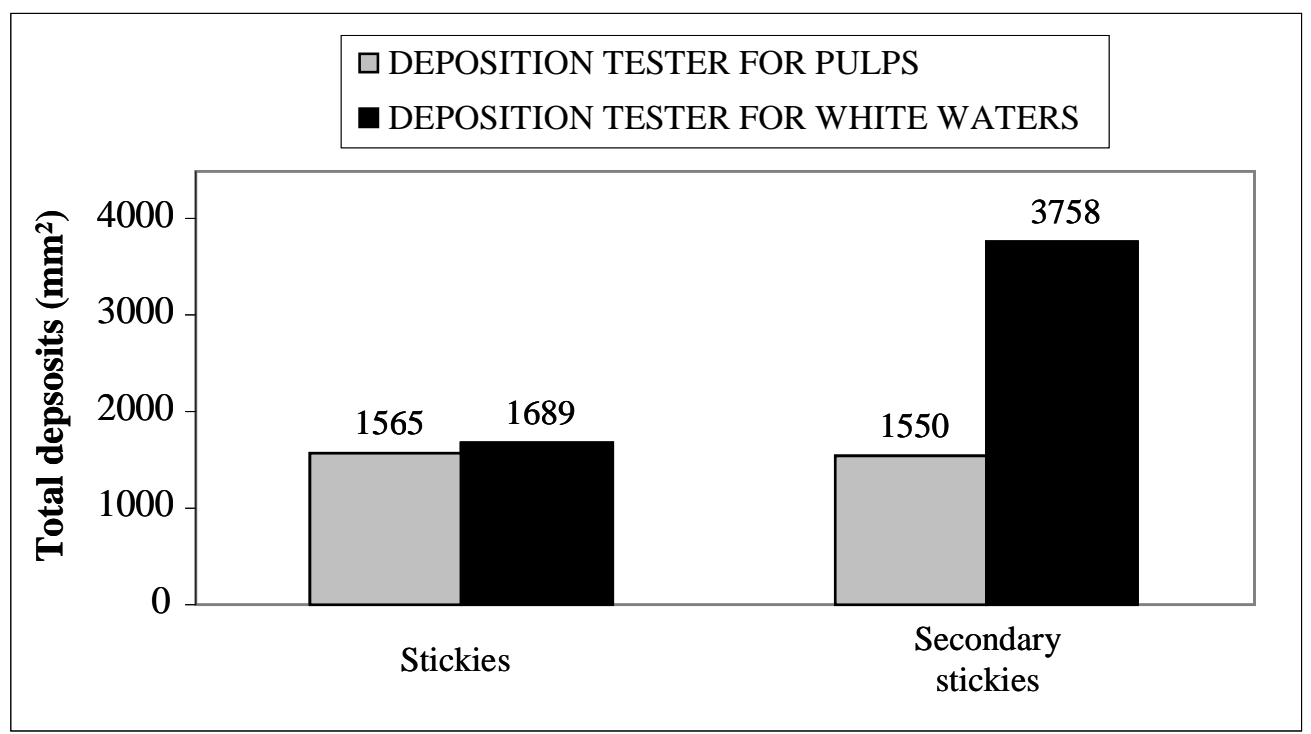

Figure 11. Comparison of the total deposits obtained by both rotors using an industrial sample

\section{Conclusions}

- A deposition tester has been designed to be directly used on pulp samples with consistencies up to $1 \%$, avoiding at the same time any filtration or dilution stages. It is possible for the improved tester to study the total stickies and the potential of DCM in pulps, to destabilise and finally to form secondary stickies with good repeatability and an error around 6\%.

- As with the deposition tester for white waters, the new device has various advantages, such as:

- $\quad$ Easy to handle and to control the applied parameters;

- It is possible to study collision and transference deposition mechanisms;

- It differentiates between sticky materials and non-sticky materials;

- It is possible to quantify the deposits by using image analysis;

- If necessary, the deposits can be extracted for further analysis. 
- The improved deposition tester was successfully validated by studying the destabilisation of different types of DCM

- The quantity of the deposits obtained with the modified device is slightly smaller than the deposits obtained with the tester for white waters due to the presence of fibres in the sample. The quantity of secondary stickies is also smaller, due to fixation of the deposited material onto the fibres by the destabilization agent.

- In general, the results taken with the improved deposition tester are similar in value, which enables it to be able to estimate the content of the stickies of the samples when filtration and/or dilution steps are not possible.

\section{Abbreviations}

$\begin{array}{ll}\text { CI } & \text { Confidence interval } \\ \text { DCM } & \text { Dissolved and colloidal material } \\ \text { DDJ } & \text { Dynamic drainage jar } \\ \text { DIP } & \text { Deinking pulp } \\ \mathrm{n} & \text { Size of the sample } \\ \text { PEI } & \text { Poly-ethylene-imine } \\ \mathrm{t} & \text { t-Student for confidence level of } 95 \% \text { and }(\mathrm{n}-1) \text { degrees of freedom } \\ \mathrm{UCM} & \text { University Complutense of Madrid } \\ \bar{X} & \text { Mean value } \\ \sigma & \text { Standard deviation of the sample }\end{array}$

\section{Acknowledgements}

The authors gratefully acknowledge funding for this research from the Community of Madrid for the Projects PROLIPAPEL I-CM (S-0505/AMB/0100) and PROLIPAPEL II-CM (S-2009/AMB/1480); from the Ministry of Science and Innovation for the Project CTM2008-06886-C02-01; and from the European Union for the AQUAFIT Project (Ref. 211534).

\section{References}

Blanco, A., García, J., Monte, M.C., Negro, C., Tijero, J., 1998a. European Patent No. 98901981.5 .

Blanco, A., Miranda, R., Negro, C., García-Súarez, C., García-Prol, M., Sánchez, A., 2007. Full characterisation of stickies in a newsprint mill: the need for a complementary approach. Tappi J., 6(1), 19-25.

Blanco, A., Negro, C., Monte, M.C., Tijero, J., 2002a. Overview of two major deposit problems in recycling: slime and stickies. Part I: slime problems in recycling. Prog. Pap. Recycling, 11(2), 14-25.

Blanco, A., Negro, C., Monte, M.C., Fuente, H., Tijero, J., 2002b. Overview of two major deposit problems in recycling: slime and stickies. Part II: stickies problems in recycling. Prog. Pap. Recycling, 11(2), 26-37. 
Blanco, A., Negro, C., Monte, M.C., Otero, D., Tijero, J., 2001. Destabilization of dissolved and colloidal material derived from coated paper. Appita J., 54(2), 132-135.

Blanco, A., Negro, C., Monte, M.C., Otero, D., Tijero, J., 2000. New system to predict deposits due to DCM destabilization in paper mills. Pulp Pap. Can., 101(9), 40-43.

Blanco, A., Negro, C., Tijero, J., 1998b. Paper recycling: An introduction to problems and their solutions, first ed. European Commision, Luxembourg.

Brun, J., Delagoutte, T., Carre, B., 2007. Origins and effects of dissolved and colloidal materials. Prog. Pap. Recycling, 17(1), 12-21.

Doshi, M.R., 1997. What is the difference between primary and secondary stickies? Prog. Pap. Recycl., 7 (1), 84-85.

Doshi, M.R., Blanco, A., Negro, C., Dorris, G.M., Castro, C.C., Hamann, A., Haynes, R.D., Houtman, C., Scallon, K., Putz, H.J., Johansson, H., Venditti, R.A., Copeland, K., Chang, H.M., 2003a. Comparison of microstickies measurement methods. Part I: sample preparation and measurement methods. Prog. Pap. Recycling, 12(4), 35-42.

Doshi, M.R., Blanco, A., Negro, C., Monte, C., Dorris, G.M., Castro, C.C., Hamann, A., Haynes, R.D., Houtman, C., Scallon, K., Putz, H.J., Johansson, H., Venditti, R.A., Copeland, K., Chang, H.M., 2003b. Comparison of microstickies measurement methods. Part II: results and discussion. Prog. Pap. Recycling, 13(1), 44-53.

Miranda, R., Monte, M.C., Blanco, A., 2011. Impact of increased collection rates and the use of commingled collection systems on the quality of recovered paper. Part 1: Increased collection rates. Waste Manag., 31 (11), 2208-2216.

Monte, M.C., 2000. Thesis, Department of Chemical Engineering, University Complutense of Madrid.

Monte, M.C., Blanco, A., Negro, C., Tijero, J., 2004. Development of a methodology to predict sticky deposits due to the destabilisation of dissolved and colloidal material in papermaking- application to different systems. Chem. Eng. J., 105, 21-29.

Monte, M.C., MacNeil, D., Negro, C., Blanco, A., 2010. Interaction of dissolved and colloidal material during the mixing of different pulps. Holzforschung, 64, 277-283.

Negro, C., Blanco, A., Monte, M.C., Otero, D., Tijero, J., 1999. Depositability character of disturbance substances. Paper Technology, 40(3), 29-34.

Ricard, M., Dorris, G., 2007a. Recirculation contaminates whitewater solids. Part I: supernatant and fines isolation and characterization. 93rd Annual Meeting of the Pulp and Paper Technical Association of Canada, Montreal, QC, Canada, 5-9 Feb., ISBN 9871897023273.

Ricard, M., Dorris, G., 2007b. Recirculation contaminates whitewater solids. Part II: contamination of fines and fillers by extractives and metals. 93rd Annual meeting of the 
Pulp and Paper Technical Association of Canada, Montreal, QC, Canada, 5-9 Feb., ISBN 9871897023273.

Sundberg, A., Ekman, R., Holmbom, B., Sundberg, K., Thornton J., 1993. Interactions between dissolved and colloidal substances and a cationic fixing agent in mechanical pulp suspensions. Nord. Pulp Pap. Res. J., 8(1), 226-231.

Tijero, A., Monte, M.C., Tijero, J. , Blanco, A., 2011. Pitch detackification with natural and modified talcs. Tappi J., 10 (10), 53-59. 


\section{Captions to figures}

Figure 2. Effect of adhesive concentration on the deposit formation at $0.5 \%$ consistency Figure 3. Effect of adhesive concentration on the deposit formation at $1 \%$ consistency Figure 4. Deposits obtained at 1\% consistency pulp

Figure 5. Effect of adhesive concentration on the secondary stickies formation at $0.5 \%$ consistency

Figure 6. Effect of adhesive concentration on the secondary stickies formation at $1 \%$ consistency

Figure 7. Effect of contaminant concentration on the deposit formation at $1 \%$ consistency

Figure 8. Comparison of the external and internal deposition obtained by both testers at different pulp consistencies

Figure 9. Comparison of the total deposits obtained by both testers at different pulp consistencies

Figure 10. Comparison of the external and internal deposition obtained by both deposition testers using an industrial sample

Figure 11. Comparison of the total deposits obtained by both testers using an industrial sample 